\title{
A rare but treatable inborn error of metabolism: Arginine:glycine amidinotransferase (AGAT) deficiency
}

\author{
Sebastian Romeo Pintilie', Adriana Fodor ${ }^{2,3}$, Marius Bembea ${ }^{4,5}$, Codruta Diana Petchesi ${ }^{4}$, \\ Simona Grad ${ }^{6,7}$, Laura Damian ${ }^{8,9}$, Romana Vulturar ${ }^{10,11}$ \\ ${ }^{1}$ Faculty of Medicine, "Iuliu Hatieganu" University of Medicine and Pharmacy, Cluj-Napoca, Romania \\ ${ }^{2}$ Clinical Center of Diabetes, Nutrition and Metabolic Diseases, Cluj-Napoca, Romania \\ ${ }^{3}$ Department of Diabetes and Eating Disorders, "Iuliu Hatieganu" University of Medicine and Pharmacy, \\ Cluj-Napoca, Romania \\ ${ }^{4}$ Department of Genetics, Faculty of Medicine and Pharmacy, University of Oradea, Romania \\ 5"Dr. Gavril Curteanu" Municipal Clinical Hospital, Regional Center for Medical Genetics Bihor, \\ Oradea, Romania \\ ${ }^{6}$ Department of Internal Medicine, "Iuliu Hatieganu" University of Medicine and Pharmacy, \\ Cluj-Napoca, Romania \\ ${ }^{7} 2^{\text {nd }}$ Department of Internal Medicine, Emergency Clinical County Hospital Cluj, Cluj-Napoca, Romania \\ ${ }^{8}$ Department of Rheumatology, Center for Rare Autoimmune and Autoinflammatory Diseases, \\ Emergency Clinical County Hospital Cluj, Cluj-Napoca, Romania \\ ${ }^{9} \mathrm{CMI}$ Reumatologie Dr. Damian, Cluj-Napoca, Romania \\ ${ }^{10}$ Department of Molecular Sciences, "Iuliu Hatieganu" University of Medicine and Pharmacy, \\ Cluj-Napoca, Romania \\ ${ }^{11}$ Cognitive Neuroscience Laboratory, "Babes-Bolyai“ University Cluj-Napoca, Romania
}

AGAT deficiency is a rare and treatable autosomal recessive disorder. The symptoms are early-onset developmental mild to moderate intellectual disability, delayed speech acquisition, behavioral problems or proximal muscle weakness. Biochemical screening for creatine, creatinine and urinary guanidinoacetate and genetic tests are used for diagnosis. Electromyography may be normal or may have a myopathic pattern with low amplitude polyphasic waves. Muscle biopsy may show abnormalities including small myocytes. Creatine supplementation can fully prevent the neurological disability, if the treatment is started early in life; the muscular function improves irrespective of the supplementation moment.

Keywords: AGAT, GAMT, CRTR, creatine deficiency syndromes, intellectual disability, language delay, creatine-phosphocreatine system

\section{INTRODUCTION}

L-arginine:glycine amidinotransferase (AGAT) deficiency, or AGAT-d, is an inborn error of metabolism that, along with guanidinoacetate methyltransferase (GAMT) deficiency and creatine transporter
(CRTR) deficiency, affect the creatine metabolism and are known as creatine deficiency $(1,2)$.

AGAT has a 5-fold pseudosymmetry of circularly arranged $\beta \beta a \beta$-modules, with an overall structure resembling a "basket with handles" (4). The enzyme 


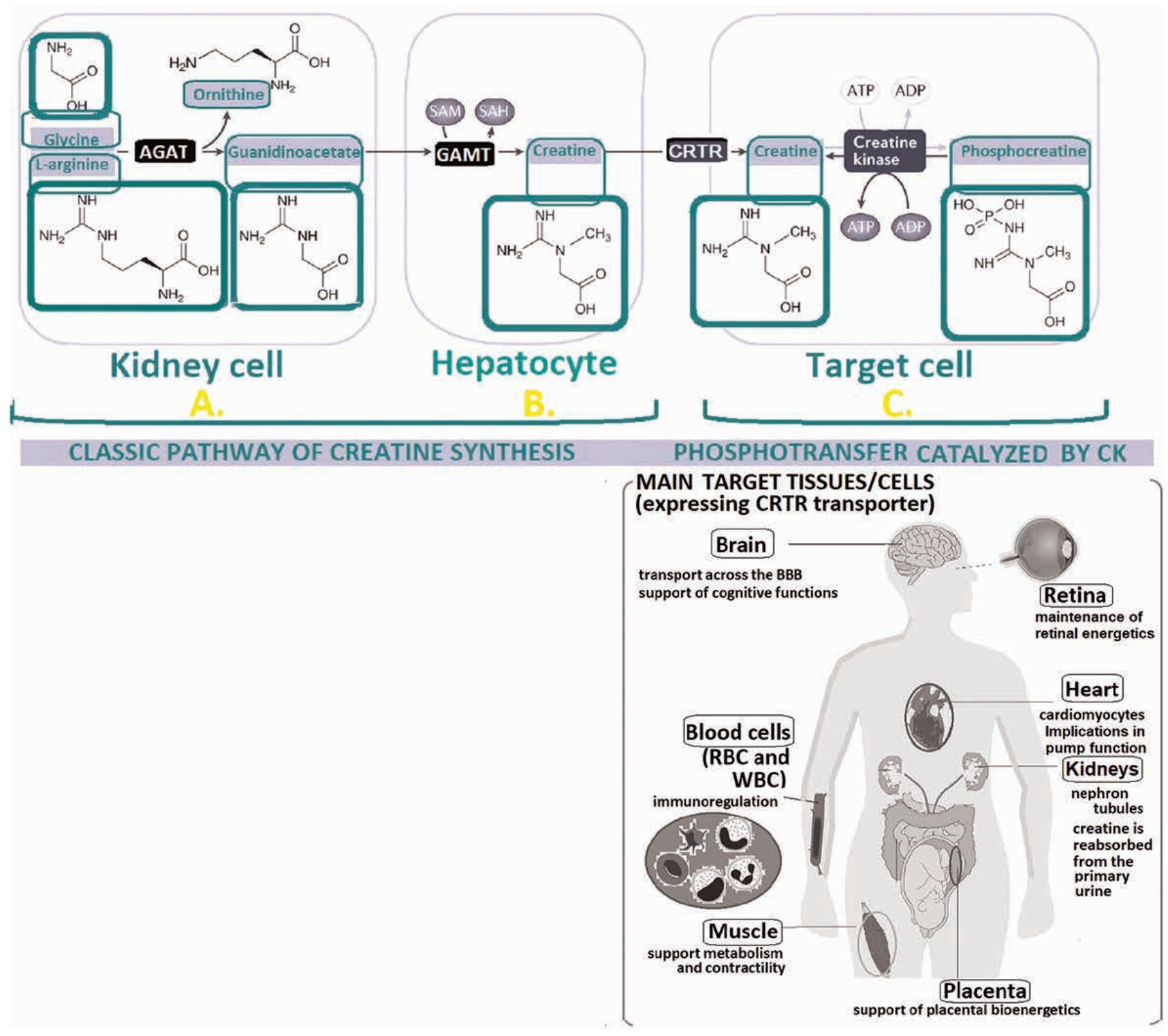

FIGURE 1. Representation of the endogenous creatine synthesis and transport of creatine into the main tissues and cells incapable of self-synthesizing sufficient amounts of creatine, but expressing the CRTR transporter (i.e., brain, muscle, blood cells, skeletal muscle, retina, heart, kidney, placenta etc) - adapted after (3).

A. In the kidney cells, synthesis of guanidinoacetate with enzyme AGAT: arginine:glycine amidinotransferase;

B. Guanidinoacetate is exported to the circulation and taken up by hepatic cells, where guanidinoacetate $\mathrm{N}$-methyltransferase (GAMT) converts it to creatine. C. Creatine is released from hepatocytes into the circulation, and is taken by cells expressing the creatine transporter (CRTR or SLC6A8). Creatine can be phosphorylated to phosphocreatine by creatine kinase, using ATP. During times of high and fluctuating energy demand (high ATP consumption), phosphocreatine can be used in reverse by creatine kinase to buffer the ATP pool. SAH: S- adenosylhomocysteine; SAM: S-adenosylmethionine, BBB: blood-brain barrier, RBC: red blood cells, WBC: white blood cells

is mainly found in the cortex of the kidney, where it catalyzes the transfer of an amidino group from Larginine to glycine, therefore producing guanidinoacetic acid (GAA) and L-ornithine (5), though L-homoarginine can also be produced $(4,6)$. In man, AGAT is also found in pancreas, liver, muscle, brain and possibly in heart $(7,8)$. Sub-cellularly, it is localized in the mitochondria intermembrane space (where it has 386 aminoacids) and to a lesser extent in cytoplasm (with 391 aminoacids) (9).
AGAT catalyzes the first step in the creatine biosynthesis pathway. The resulting guanidinoacetic acid is transported to the liver, where it is methylated to creatine by GAMT $(5,10)$. Creatine is then transported to the tissues that require it (such as neurons, myocytes and cardiomyocytes) where the uptake is regulated by special creatine transporter (SLC6A8). There, it is involved in energy storage and transmission through its involvement in ATP (adenosine triphosphate) production (11). 
In the neuron, the creatine/phosphocreatine system helps couple ATP-consuming with ATP-producing processes (12). SLC6A8 transporter allows creatine to penetrate the brain-blood barrier in modest amounts $(13,14)$. Most brain cells express only one or none of the creatine biosynthesis pathway enzymes $(13,15)$. In consequence, the brain is almost totally dependent on the uptake of exogenous creatine or endogenous creatine produced in other tissues.

Most creatine is found nevertheless in skeletal muscle, where it plays a similar role (11). About $1.7 \%$ of the total creatine content of a human is converted daily to creatinine and excreted in urine, i.e. two grams of creatine per day for a 70-kg man (5). When AGAT is not functional, the only option for creatine replenishment is the uptake of creatine from alimentation (16).

The usual symptoms of AGAT-d are developmental and language delay, intellectual disability, behavioral problems, myopathy or proximal muscle weakness (17). Rarely, seizures can appear. This is in contrast to GAMT deficiency (the second step in the creatine synthesis - Figure 1), where the buildup of guanidinoacetic acid has neurotoxic effects (15) and frequently is manifested with seizures (11).

\section{GENETICS}

AGAT deficiency is an autosomal recessive disorder. The AGAT gene (official nomenclature GATM, encoding a mitochondrial enzyme that belongs to the amidinotransferase family) has been mapped to chromosom region $15 q$ and contains nine exons. Nine different pathogenic mutations (nonsense, splice, frame shift, missense) have been reported so far (2). Both nonsense and missense mutations of the GATM gene can lead to the enzyme loss of function (17); normally this enzyme catalyzes the first step in the creatine biosynthesis pathway - Figure 1 $(5,9)$.

Mutations in the gene coding the creatine transporter SLC6A8 or CRTR lead to CRTR deficiency, with symptoms similar to AGAT-d, but much harder to treat; in some cases, folding defects are amenable to rescue by small molecules like pharmacochaperones and/or allosteric modulators (18). AGAT deficiency might represent the most underdiagnosed disease among the creatine deficiency syndromes, and has the mildest clinical phenotype comparing with GAMT or CRTR deficiencies (2).

\section{PATHOPHYSIOLOGY}

The lack of AGAT disrupts the entire creatine biosynthesis pathway, leading to a lack of endogenous creatine. This affects tissues with high and fluctuat- ing energy demands which usually contain the highest levels of creatine, such as brain, skeletal and heart muscle, but also photoreceptor cells of the retina and spermatozoa, although no ophthalmological or sexual function-related symptoms of AGAT-d have been described so far $(2,5)$.

Besides the role in the energetic metabolism, creatine is also relevant in GABAergic and glutamatergic signaling in the central nervous system, has antioxidant properties and helps water retention in muscle (9). Creatine also plays a role in certain gene transcription regulation in brain. Two genes, BCAS1 and SLC6A8, upregulated in AGAT-d mice, normalize their expression after creatine supplementation (19). Bcas1 is an important protein found in brain whose level increases after stroke, most likely as a compensatory mechanism to energy deficiency and hypoxia (20), and whose decrease is associated with hypomyelination (19). SLC6A8 encoding the creatine transmembrane transporter (CRTR- Figure 1), is upregulated in AGAT-d mice probably as a compensatory mechanism $(13,19)$. The expression of SLC6A8 on the brain-blood barrier (BBB) allows the entry of creatine especially in early life, when the BBB is not as tightly regulated as in maturity, partly explaining the efficacy of early, pre-symptomatic treatment $(9,14)$. AGAT-d mice deficient behavior and spatial memory improved after creatine supplementation (21).

Creatine deficiency also alters muscle energy metabolism, where the creatine-phosphocreatine system helps couple oxidative phosphorylation with the ADP-ATP system (22,23). In AGAT-d mice, the lack of creatine was associated with muscular atrophy, smaller myocyte diameter, and an increased dependence on glycolytic ATP production, due to the dysfunction of mitochondrial respiratory chain complexes - all reversed by creatine supplementation (22). Creatine levels in muscle reached normal levels in one day, while hypothalamic or hippocampal levels took 20 days to normalize (22). Mice AGAT-d muscle also presented more than one hundred significantly regulated genes implicated in pyruvate and glucose metabolism, oxidation/reduction, and one-carbon metabolism (23). In conclusion, in the absence of creatine, skeletal muscle is severely affected (24).

Creatine is also found in spermatozoa (5) and AGAT-d mice have been found to be sterile (9). However, creatine kinase function in spermatozoa may not correlate with male fertility, since energy can also be produced by glycolysis, independent of creatine kinase (25). This calls for further research in the relationship between creatine and reproductive system health.

Since creatine is involved in multiple metabolic processes, AGAT-d mice can exhibit abnormal meta- 
bolic phenotypes (23). Creatine-deficient mice present enhanced glucose tolerance, reduced adiposity and cholesterol levels, due to the chronic creatinedependent activation of AMP-activated protein kinase (26). Moreover, the expression of GLUT4 is elevated, and hepatic gluconeogenesis is severely impaired (23).

Apart from guanidinoacetic acid, AGAT can also synthesize L-homoarginine by transferring the amidino group to lysine instead of glycine (4). Low levels of homoarginine are associated with heart failure increased cardiovascular risk and mortality (4, 6). In heart failure the expression and enzyme activity of AGAT are increased (8). On the other hand, homoarginine acts as a weak substrate for nitric oxide synthase (NOS) (6) and high levels of homoarginine may subsequently increase NOS activity and blood pressure (4). Homoarginine supplementation also restores the expression of Kcnip3 and Ivd, two genes relevant to cerebrovascular function, downregulated in AGAT-d mice (19). The first codes for calsenilin, a neuronal calcium-binding protein implicated in Alzheimer's disease (27). The second codes for an enzyme implicated in L-leucine degradation, and in consequence, AGAT-d murine brains show increased leucine levels, which did not normalize with homoarginine supplementation (19). In healthy volunteers who were administered creatine, circulating homoarginine increased by $35 \%$ (28). This study could suggest long-term cardiovascular implications of daily creatine supplementation in AGAT-d patients (4).

AGAT might also be indirectly linked to gyrate atrophy of the choroid and retina, another rare inherited disease caused by the deficiency of ornithine aminotransferase, which leads to ornithine concentrations 10 to 20 times those found in normal plasma, and also to creatine deficiency $(7,29)$. Since ornithine is an inhibitor of AGAT, increased circulating ornithine might suppress creatine synthesis through AGAT inhibition, which would lead to a lack of readily available energy, and thus atrophy (7). No opthalmic symptoms have been reported in AGAT-d patients so far, but further research is necessary (17).

TABLE 1. Physiology and pathophysiology in AGAT-related creatine metabolism

\begin{tabular}{|c|c|c|}
\hline System involved & $\begin{array}{l}\text { Clinical and molecular features affected } \\
\text { by AGAT-related creatine metabolism }\end{array}$ & References \\
\hline \multirow{6}{*}{ Brain } & Signaling in GABAergic and glutamatergic pathways in the central nervous system & (9) \\
\hline & $\begin{array}{l}\text { Regulations of several genes involved in compensatory mechanisms after stroke (energy deficiency } \\
\text { and hypoxia), myelination }\end{array}$ & (19) \\
\hline & In AGAT-d mice, the behavior and spatial memory is improved with creatine supplementation & $(21)$ \\
\hline & Synthesis of calsenilin, a neuronal calcium-binding protein involved in Alzheimer's disease & $(27)$ \\
\hline & Influences the expression of SLC6A8 gene on the brain-blood barrier & $(9,14,19)$ \\
\hline & $\begin{array}{l}\text { Affect the expression of calsenilin, a neuronal calcium-binding protein implicated in Alzheimer's } \\
\text { disease, involved in AGAT-d mice }\end{array}$ & $(19,27)$ \\
\hline Eye & $\begin{array}{l}\text { Likely indirectly implications in gyrate atrophy of the choroid and retina ornithine due to the high } \\
\text { ornithine levels that suppress creatine synthesis through AGAT inhibition; secondary this will cause } \\
\text { lack of energy and atrophy }\end{array}$ & $(7,29)$ \\
\hline \multirow{5}{*}{ Muscle } & $\begin{array}{l}\text { Implicated in buffering reactions in mitochondrial ADP-ATP system, affects the muscle energy } \\
\text { metabolism }\end{array}$ & $(22,23)$ \\
\hline & Antioxidant properties & (9) \\
\hline & Stimulates water retention in muscle & (9) \\
\hline & $\begin{array}{l}\text { Normal development of myocytes; lack of creatine in AGAT-d mice is associated with muscular } \\
\text { atrophy, smaller myocyte diameter, increased dependence on glycolytic ATP production }\end{array}$ & $(22)$ \\
\hline & $\begin{array}{l}\text { Gene regulation in AGAT-d mice related to pyruvate and glucose metabolism, oxidation/reduction } \\
\text { reactions, one-carbon metabolism }\end{array}$ & $(23)$ \\
\hline \multirow{4}{*}{ Metabolism } & $\begin{array}{l}\text { Energetic metabolism, physiology of mitochondrial respiratory chain complexes coupled with ADP- } \\
\text { ATP system }\end{array}$ & $(22,23)$ \\
\hline & Affects the expression of GLUT4 gene & $(23)$ \\
\hline & $\begin{array}{l}\text { Influences glucose tolerance; hepatic gluconeogenesis is severely impaired in creatine-deficient } \\
\text { mice }\end{array}$ & $(23)$ \\
\hline & $\begin{array}{l}\text { Involvement in adiposity and cholesterol levels through the chronic creatine-dependent activation } \\
\text { of AMP-activated protein kinase }\end{array}$ & $(26)$ \\
\hline \multirow{2}{*}{$\begin{array}{l}\text { Cardio-vascular } \\
\text { system }\end{array}$} & Implications in heart pump function through low synthesis of L-homoarginine & $(4,6)$ \\
\hline & Affects NO (nitric oxide) metabolism and blood pressure through synthesis of L-homoarginine & $(4,6)$ \\
\hline $\begin{array}{l}\text { Reproductive } \\
\text { system }\end{array}$ & Creatine is also found in spermatozoa; there are possible implications in sterility ins AGAT- $d$ mice & $(5,9,25)$ \\
\hline
\end{tabular}




\section{DIAGNOSTIC AND SCREENING}

AGAT-d can be diagnosed by measuring blood and urine guanidinoacetate, creatine and creatinine levels. Plasma and urine guanidinoacetate in AGAT-d are undetectable, or $<10 \%$ of lower range of the reference values. Plasma amino acid concentrations (excepting S-adenosyl-homocysteine, SAH) are normal in AGAT-d (17). Newborn biochemical screening for AGAT-d is challenging, as the normal range for guanidinoacetic acid is below the detection limit of assays suitable for blood spot analysis. The GATM gene can be sequenced and were identified several types of mutations (11). Brain creatine levels can be measured using proton magnetic resonance spectroscopy ( $\mathrm{H}^{1}$-MRS), and is used mostly for monitoring the treatment (2).

\section{CLINICAL PICTURE}

The main characteristic of AGAT-d is intellectual disability, as creatine has been associated with the development of higher cognitive functions (14). Most patients had language delay (with onset during the first year of life), behavioral problems and mild to moderate mental retardation, with intelligence or developmental quotients (IQ/DQ) between 35 and 60 before treatment $(2,16,17)$. In the absence of creatine, irreversible brain damage may occur in the first year of life $(14,17,30)$. Oral creatine treatment marginally improves IQ/DQ scores in humans despite almost full brain creatine replenishment in older patients, but the earlier treatment was started, the greater recovery in brain function was attained (3). Cerebral MRI shows normal brain anatomy and structure, while in vivo brain $\mathrm{H}^{1}$-MRS reveals profound cerebral creatine deficiency $(2,16,17)$.

Myopathy, with low muscle mass and proximal muscle weakness, was reported in half of the patients with ages between 6 and 23 (17). Myopathy seems to be a symptom mostly in later age, since patients diagnosed at a young age presented no muscle weakness and developed normally following treatment (31). The electromyography (EMG) was normal or showed a myopathic pattern with low amplitude polyphasic waves $(17,23,24)$. Muscle biopsy may show small myocytes and other abnormalities, including mitochondrial at ultrastructural studies (17).

\section{TREATMENT AND PROGNOSIS}

Early diagnosis and treatment may prevent intellectual disability and myopathy in patients with AGAT-d. The treatment purpose is to restore cerebral and muscular creatine levels; the recommended doses of creatine monohydrate are 100-800 mg/ $\mathrm{kg} /$ day, and results in almost complete restoration of brain creatine levels and significant improvement of myopathy in most patients (2). Although the brain creatine levels normalizes in patients treated later, some irreversible neuronal damage occur in the first year of life $(17,14,31)$. Muscle improvement after creatine supplementation is fast, while the neurological one is slow (22).

As some metabolic disturbances were registered (such as enhanced glucose tolerance, reduced adiposity, reduced cholesterol levels, and decreased of hepatic gluconeogenesis), the metabolic parameters should be monitored during the creatine therapy $(3,23,26)$. Also, weight gain as a side effect of creatine supplementation should be considered in some treated AGAT-d patients $(23,31)$.

\section{CONCLUSIONS}

AGAT-d is a rare and treatable disorder, and should be suspected and searched for as soon as possible in infants with signs of developmental delay, muscle hypotonia and intellectual disability. Creatine supplementation can fully prevent the neurological dysability, if started early in life; the muscular function improves irrespective of the supplementation moment.

\section{Acknowledgement}

The last two authors have equal contribution.

Conflict of interest: none declared Financial support: none declared

\section{REFERENCES}

1. Item CB, Stoöckler-Ipsiroglu S, Stromberger C, et al. Arginine:Glycine amidino-transferase deficiency: The third inborn error of creatine metabolism in humans. Am J Hum Genet. 2001;69(5):1127-1133.

2. Stockler-Ipsiroglu S, Mercimek-Mahmutoglu S, Salomons GS. Creatine deficiency syndromes. In: Saudubray, J.M., Baumgartner, M.R., Walter J. (eds) Inborn Metabolic Diseases: Diagnosis and Treatment. 6th ed. Springer, 2016;243-250.

3. Kazak L, Cohen P. Creatine metabolism: energy homeostasis, immunity and cancer biology. Nat Rev Endocrinol. 2020;16(8):421-436.
4. Davids M, Ndika JDT, Salomons GS, Blom HJ, Teerlink T. Promiscuous activity of arginine: Glycine amidinotransferase is responsible for the synthesis of the novel cardiovascular risk factor homoarginine. FEBS Lett. 2012;586(20):3653-3657.

5. Wyss M, Kaddurah-Daouk R. Creatine and creatinine metabolism. Physiol Rev. 2000;80(3):1107-1213.

6. Atzler D, Schwedhelm E, Choe C. L-Homoarginine and cardiovascular disease. Curr Opin Clin Nutr Metab Care. 2015;18(1):83-88. 
7. Sipilä I. Inhibition of arginine-glycine amidinotransferase by ornithine. A possible mechanism for the muscular and chorioretinal atrophies in gyrate atrophy of the choroid and retina with hyperornithinemia. BBA - Enzymol. 1980;613(1):79-84.

8. Cullen ME, Yuen AH, Felkin LE, Smolenski RT, Hall JL, Grindle S, Miller LW, Birks EJ, Yacoub MH, Barton PJ. Myocardial expression of the arginine:glycine amidino-transferase gene is elevated in heart failure and normalized after recovery: potential implications for local creatine synthesis. Circulation. 2006;114 (Suppl 1):16-20.

9. Joncquel-Chevalier Curt M, Voicu PM, Fontaine M, et al. Creatine biosynthesis and transport in health and disease. Biochimie. 2015; 119:146-165.

10. Tachikawa M, Ikeda S, Fujinawa J, Hirose S, Akanuma S, Hosoya K. $Y$-Aminobutyric acid transporter 2 mediates the hepatic uptake of guanidinoacetate, the creatine biosynthetic precursor, in rats. PLOS One. 2012;7(2):e32557.

11. Nasrallah F, Feki M, Kaabachi N. Creatine and Creatine Deficiency Syndromes: Biochemical and Clinical Aspects. Pediatr Neurol. 2010; 42(3):163-171.

12. Ames A. CNS energy metabolism as related to function. Brain Res Rev. 2000;34(1-2):42-68.

13. Braissant O, Henry H. AGAT, GAMT and SLC6A8 distribution in the central nervous system, in relation to creatine deficiency syndromes: a review. J Inherit Metab Dis. 2008;31(2):230-9.

14. Braissant 0 . Creatine and guanidinoacetate transport at blood-brain and blood-cerebrospinal fluid barriers. J Inherit Metab Dis. 2012;35(4):655-64.

15. Ostojic SM. Benefits and drawbacks of guanidinoacetic acid as a possible treatment to replenish cerebral creatine in AGAT deficiency. Nutr Neurosci. 2019;22(5):302-305.

16. Battini R, Alessandrì MG, Leuzzi V, et al. Arginine:glycine amidinotransferase (AGAT) deficiency in a newborn: Early treatment can prevent phenotypic expression of the disease. J Pediatr. 2006;148(6):828-830.

17. Stockler-Ipsiroglu S, Apatean D, Battini R, et al. Arginine: Glycine amidinotransferase (AGAT) deficiency: Clinical features and long term outcomes in 16 patients diagnosed worldwide. Mol Genet Metab. 2015;116(4):252-259.

18. Farr CV, El-Kasaby A, Freissmuth M, Sucic S. The Creatine Transporter Unfolded: A Knotty Premise in the Cerebral Creatine Deficiency Syndrome. Front Synaptic Neurosci. 2020;12:588954.

19. Jensen M, Müller C, Schwedhelm E, et al. Homoarginine-and creatine-dependent gene regulation in murine brains with
I-arginine:Glycine amidinotransferase deficiency. Int J Mol Sci. 2020;21(5).

20. Dingman AL, Rodgers KM, Dietz RM, et al. Oligodendrocyte Progenitor Cell Proliferation and Fate after White Matter Stroke in Juvenile and Adult Mice. In: Developmental Neuroscience. Vol 40. S. Karger AG; 2019:601-616

21. Iqbal F, Hoeger $\mathrm{H}$, Lubec $\mathrm{G}$, Bodamer O. Biochemical and behavioral phenotype of AGAT and GAMT deficient mice following long-term Creatine monohydrate supplementation. Metab Brain Dis. 2017; 32(6):1951-1961.

22. Nabuurs $\mathrm{Cl}$, Choe $\mathrm{CU}$, Veltien $\mathrm{A}$, et al. Disturbed energy metabolism and muscular dystrophy caused by pure creatine deficiency are reversible by creatine intake. J Physiol. 2013;591(2):571-592.

23. Stockebrand M, Nejad AS, Neu A, et al. Transcriptomic and metabolic analyses reveal salvage pathways in creatine-deficient AGAT-/- mice. Amino Acids. 2016;48(8):2025-2039.

24. Snow RJ. AGAT knockout mice provide an opportunity to titrate tissue creatine content. J Physiol. 2013;591(2):393.

25. Rolf C, Behre HM, Cooper TG, Koppers B, Nieschlag E. Creatine kinase activity in human spermatozoa and seminal plasma lacks predictive value for male fertility in in vitro fertilization. Fertil Steril. 1998; 69(4):727-734

26. Choe CU, Nabuurs C, Stockebrand MC, et al. L-arginine: Glycine amidinotransferase deficiency protects from metabolic syndrome. Hum Mol Genet. 2013;22(1):110-123.

27. Jin JK, Choi JK, Wasco W, et al. Expression of calsenilin in neurons and astrocytes in the Alzheimer's disease brain. Neuroreport. 2005; 16(5):451-455

28. Derave W, Marescau B, Vanden Eede E, Eijnde BO, De Deyn PP, Hespel $P$. Plas-ma guanidino compounds are altered by oral creatine supplementation in healthy humans. J Appl Physiol. 2004; 97(3):852-857.

29. Elnahry AG, Tripathy K. Gyrate Atrophy Of The Choroid and Retina, 2020. Avalable at: http://www.ncbi.nlm.nih.gov/pubmed/32491691.

30. Bianchi MC, Tosetti M, Fornai F, et al. Reversible brain creatine deficiency in two sisters with normal blood creatine level. Ann Neurol. 2000;47(4):511-513.

31. Battini R, Alessandrì MG, Casalini C, Casarano M, Tosetti M, Cioni G. Fifteen-year follow-up of Italian families affected by arginine glycine amidinotransferase deficiency. Orphanet J Rare Dis. 2017;12(1):1-9. 